(C) 2015 IEEE. Personal use of this material is permitted. Permission from IEEE must be obtained for all other uses, in any current or future media, including reprinting/republishing this material for advertising or promotional purposes, creating new collective works, for resale or redistribution to servers or lists, or reuse of any copyrighted component of this work in other works. 


\title{
Alternative Conducted Emission Measurements On Mains Without LISNs
}

\author{
Osman Şen', Soydan Çakır', Mehmet Çınar', Marc Pouss', Ferran Silva², Mustafa Çetintas' \\ 'Electromagnetic Laboratories, TUBITAK UME, Gebze, Kocaeli, Turkey \\ 2Grup de Compatibilitat Electromagnètica (GCEM) DEE ETSETB Universitat Politècnica de Catalunya,
}

\author{
Barcelona, Spain
}

\begin{abstract}
Conducted emission tests are always performed by the use of LISNs in laboratories in accordance with CISPR22, CISPR11 and other similar standards. However, it is not always possible to use LISNs because of some limitations. If the EUT (Equipment Under Test) has large dimensions or high currents, it is not, for most of the time, possible to send it to an EMC laboratory or to use LISNs during the test. As a consequence, usage or development of alternative conducted emission test methods is inevitable in industry. In this paper, we made conducted measurements on actual EUTs in alternative environments whose impedances are different from the standard LISN impedance and continued to establish the fundamentals of alternative conducted emission tests based on the impedance measurements of the EUT, supply and used cables. We also established the correlation, on the basis of impedance measurements, between these alternative conducted emission test method and the reference conducted emission test method.
\end{abstract}

Index Terms - Alternative, Current Probe, Conducted Emission, EMC, High Current, Industry, LISN, Mains Impedance

\section{Introduction}

All equipment placed on the European Market has to fulfill the essential requirements of the European EMC Directive. The normal approach is to show compliance with basic test requirements and testing electrical and electronic products is a must before entering the market. Actually, EMC measurement and validation are necessary during the whole period of development of products. However, development, implementation and maintenance of the EMC measurement facility in accordance with standards are heavy loads for industry. Using the facility in EMC laboratories is a solution but expensive and time consuming. In addition, most of the time, it is not always possible to use standard laboratory EMC methods for some EUTs which are large, stationary or has high currents. Conducted emission test is one of the major tests for industry and widely performed in laboratories for the frequency range $150 \mathrm{kHz}$ - $30 \mathrm{MHz}$ by the use of LISNs in accordance with CISPR22 [1], CISPR11 [2] and other similar standards. However, it is not always possible to use LISNs because of some limitations. Some of alternative methods which include current clamps, capacitive foil probe Electrical Fast Transient clamps, were well studied in [3] for large EUTs. Another research on alternative measurements without LISN is given in [4] for Motor Drive Systems, which is based on calculations. Finally, a good work on alternative methods is accomplished in [5] where, in addition to direct measurement methods, an indirect method based on the circuit models was introduced in time domain. In [6], we have established the fundamentals of conducted emission tests based on the impedance measurements of the EUT, supply and used mains cable by using two-probe approach. Although there are some methods for impedance measurements in the literature, we chose the two current probe method stated in [7] for our method. In this impedance measurement method, the impedance is measured by using two current probes, network analyzer and reference known impedances. This impedance measurement method yields the value of the unknown impedance as well as the impedance of used cables. For that reason, it was found more suitable by us for conducted emission measurement purposes in comparison by the other methods. We began with firstly establishing the common mode and differential mode circuit models to use the measured impedances of the EUT, used cables and the supply. For that purpose, in [6] we utilized the RF port of a signal generator as an EUT along with a commercial reference source and tested them under the reference conditions installed with two LISNs as stipulated by the standards and also under the unknown mains conditions installed with different connection of LISNs. In [6], LISNs connected in parallel for phase in various numbers and also for neutral were used as the unknown mains that simulate an industrial environment. The main reason for simulating the mains by using various numbers of LISNs in parallel is to form ideal mains with stable impedance and without any resonances but very different from the reference LISN impedance.

In this paper, we did not only use the mains simulations installed with different connection of LISNs like we used in [6], but also we also show how to use actual 220V mains without using any LISN. Moreover, we tested actual EUTs such as a drill, a UPS (Uninterruptable Power Supply) in addition to $220 \mathrm{~V}$ reference source on mains simulations and also on actual mains, and compared theoretical correction factors with experimental correction factors to validate the alternative method based on the impedance measurements. Finally, the CM and DM emissions measured in alternative setups are merged and linked to reference LISN results.

\section{Theory And Experimental Setup}

The proposed alternative conducted emission measurement method is completely based on the separate impedance measurements 
of the EUT, used cables and supply. The impedance measurement that we used is stated in [7] in detail. This impedance measurement method uses a network analyzer, two current probes and precision known impedance. It yields the value of the unknown impedance as well as the impedance of used cables that include the effects of the used current probes and, if any, other measurement components.

Emissions coming from an EUT are classified as CM (Common Mode) and DM (Differential Mode) and measured in laboratory environment with the use of LISNs. The circuit models of conducted emission measurements for CM and DM in laboratory environment are presented in Fig.1.
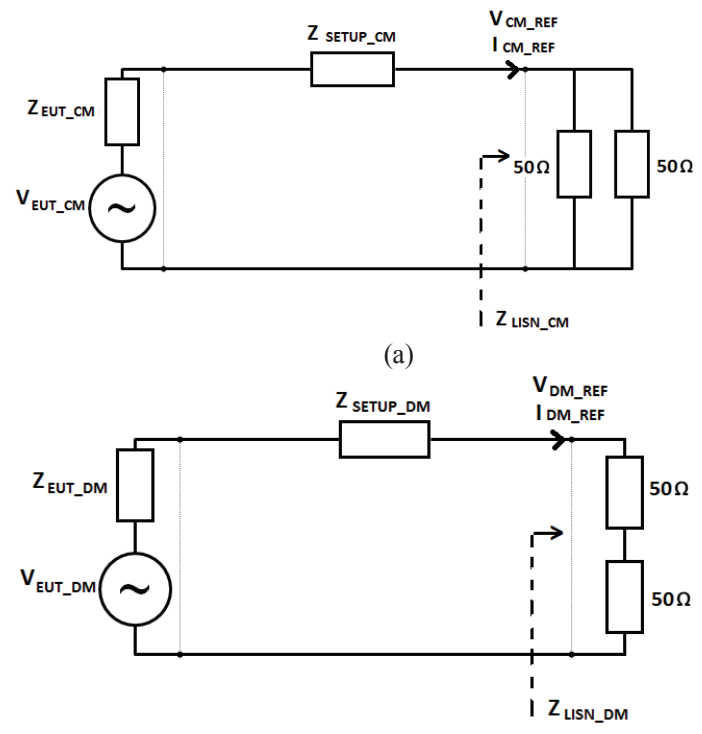

(b)

Fig 1. Circuit models of conducted emission measurements in laboratory environment (a) CM circuit model, (b) DM circuit model

As seen in Fig.1, the interference sources inside the EUT are indicated as $\mathrm{V}_{\text {EUT_CM }}$ and $\mathrm{V}_{\text {EUT_DM }}$ for CM and DM circuit models. $Z_{\text {EUT_CM }}$ and $\bar{Z}_{\text {EUT_DM }}$ are the internal impedances of the EUT. $Z_{\text {SETUP_CM }}$ and $Z_{\text {SETUP_DM }}$ are the impedances of the used cables including used measurement components such as current probes and so on. These figures show the reference setup installed with two LISNs in laboratory environment. The impedance of each used LISN is depicted as $50 \mathrm{ohm}$. The each LISN impedance becomes parallel in the CM circuit model and series in the DM model [4]. The flowing CM current and the induced CM voltage just at the LISN system in Fig.1(a) are depicted as $\mathrm{I}_{\mathrm{CM} \text { REF }}$ and $\mathrm{V}_{\mathrm{CM} \text { REF }}$ respectively for the CM model. These CM current and CM voltage can be easily calculated as given in (1) and (2). Likewise, for reference DM model, the flowing DM current and the induced DM voltage just at the LISN system in Fig.1(b) is depicted as $I_{D M}$ REF and $V_{D M}$ REF respectively. $I_{D M}$ REF and $V_{\text {DM_REF }}$ are simply calculated as given in (3) and (4). It must be specially emphasized that the impedances given in all the equations in this paper are complex numbers, consequently resultant voltages, currents and correction factors become complex numbers. However, all the graphs given in this paper only include the magnitudes of complex quantities.

$$
\begin{aligned}
& I_{C M_{-} R E F}=\frac{V_{E U T_{-} C M}}{Z_{E U T_{-} C M}+Z_{S E T P_{-} C M}+Z_{L I S N_{-} C M}} \\
& V_{C M_{-} R E F}=\frac{Z_{L I S N_{-} C M} * V_{E U T_{-} C M}}{Z_{E U T_{-} C M}+Z_{S E T U P_{-} C M}+Z_{L I S N_{-} C M}} \\
& I_{D M_{-} R E F}=\frac{V_{E U T_{-} D M}}{Z_{E U T_{-} D M}+Z_{S E T U P_{-} D M}+Z_{L I S N_{-} D M}} \\
& V_{D M_{-} R E F}=\frac{Z_{L I S N_{-} D M} * V_{E U T_{-} D M}}{Z_{E U T_{-} D M}+Z_{S E T U P_{-} D M}+Z_{L I S N_{-} D M}}
\end{aligned}
$$

On the other hand, unlike the laboratory environment, the alternative methods for industry have the circuit model shown in Fig.2.

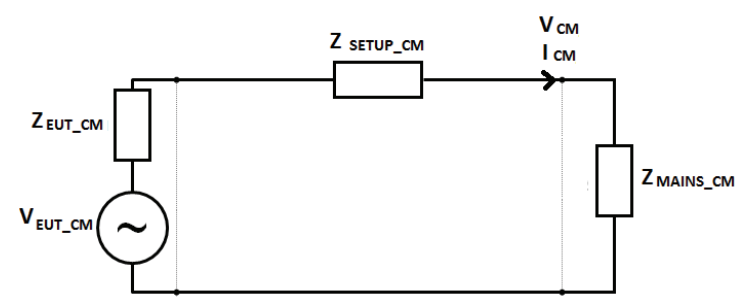

(a)

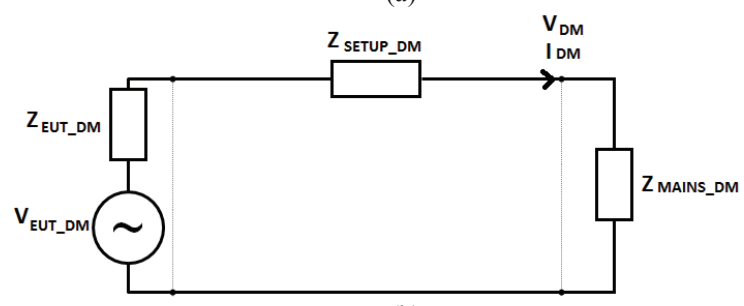

(b)

Fig 2. Circuit models of alternative conducted emission measurements in industry (a) CM circuit model, (b) DM circuit model

Currents and voltages are based on the impedance of mains instead of the reference LISN impedance, so that the equations for industry can be given as follows in (5) - (8) for CM and DM current and voltages.

$$
\begin{aligned}
I_{C M} & =\frac{V_{E U T_{-} C M}}{Z_{E U T_{-} C M}+Z_{S E T U P_{-} C M}+Z_{M A I N S_{-} C M}} \\
V_{C M} & =\frac{Z_{M A I N S_{-} C M} * V_{E U T_{-} C M}}{Z_{E U T_{-} C M}+Z_{S E T U P_{-} C M}+Z_{M A I N S_{-} C M}} \\
I_{D M} & =\frac{V_{E U T_{-} D M}}{Z_{E U T_{-} D M}+Z_{S E T U P_{-} D M}+Z_{M A I N S_{-} D M}} \\
V_{D M} & =\frac{Z_{M A I N S_{-} D M} * V_{E U T \_D M}}{Z_{E U T_{-} D M}+Z_{S E T U P_{-} D M}+Z_{M A I N S_{-} D M}}
\end{aligned}
$$

As seen in the equations (5) - (8), the only difference between the reference setup and the alternative setup is the mains impedance instead of the reference LISN impedance for both CM and DM. Besides, in the equations, the $\mathrm{CM}$ and $\mathrm{DM}$ voltages/currents are stated with only CM and DM subscripts without "REF" in order to emphasize that these are industrial currents not the reference one of the laboratory environment. Finally, all the impedance measurements lead to correction factors between the reference emission setup with the reference LISN and the alternative setup without 
the reference LISN as given in equations (9) - (12) with the assumption that $\mathrm{CM}$ and $\mathrm{DM}$ interference voltage sources inside the EUT are constant [6].

$$
\begin{aligned}
& K_{\text {Current_CM }}=\frac{I_{C M \_R E F}}{I_{C M}}=\frac{Z_{\text {EUT_CM }}+Z_{\text {SETUP_CM }}+Z_{M A I N S_{-} C M}}{Z_{\text {EUT_CM }}+Z_{\text {SETUP_CM }}+Z_{\text {LISN_CM }}} \\
& K_{\text {Current } \_D M}=\frac{I_{D M \_R E F}}{I_{D M}}=\frac{Z_{E U T_{-} D M}+Z_{S E T U P \_D M}+Z_{M A I N S_{-} D M}}{Z_{E U T_{-} D M}+Z_{S E T U P_{-} D M}+Z_{\text {LISN_DM }}} \\
& K_{\text {Voltage_CM }}=\frac{V_{C M_{-} R E F}}{V_{C M}}=K_{\text {Current_CM }_{-}} \cdot \frac{Z_{L_{\text {LISN_CM }} C M}}{Z_{M A I N S_{-} C M}} \\
& K_{\text {Voltage_DM }}=\frac{V_{D M_{-} R E F}}{V_{D M}}=K_{\text {Current_DM }_{-}} \cdot \frac{Z_{L_{I S N_{\_} D M}}}{Z_{M A I N S_{-} D M}}
\end{aligned}
$$

These factors are very essential and expected to form correlation between the laboratory and the industry. If the LISN usage is not possible due to some restrictions, measurement of the current $\left(\mathrm{I}_{\mathrm{DM}}\right.$ and $\left.\mathrm{I}_{\mathrm{CM}}\right)$ with a current clamp or measurement of the voltage $\left(V_{C M}\right.$ and $\left.V_{D M}\right)$ with a CVP is the only way to perform the measurement in industrial environment. However these measured values do not make any sense without knowing the impedances of the EUT, used cables and supply, for that reason the correction factors obtained in (9) - (10) for current and in (11) - (12) for the voltage will form the connection between the reference conducted emission method and alternative conducted emission methods. After calculating the theoretical factors based on the impedance measurements, $\mathrm{I}_{\mathrm{DM}}$ and $\mathrm{I}_{\mathrm{CM}}$ (or $\mathrm{V}_{\mathrm{DM}}$ and $\mathrm{V}_{\mathrm{CM}}$ ) measured in industry are linked to $\mathrm{I}_{\mathrm{DM} \_ \text {REF }}$ and $\mathrm{I}_{\mathrm{CM} \_ \text {REF }}\left(\mathrm{V}_{\mathrm{DM} \_ \text {REF }}\right.$ and $\left.\mathrm{V}_{\mathrm{CM} \mathrm{C}_{-} \mathrm{REF}}\right)$ in the reference setup and finally the voltage measured at the RF port of one of LISNs is predicted as given in (13) as the worst case. In (13), $Z_{\text {SINGLE_LISN }}$ is the impedance of a single LISN.

$$
\left|V_{\text {LISN }}\right|=\left(\frac{\left|I_{C M_{-} R E F}\right|}{2}+\left|I_{D_{M_{-} R E F}}\right|\right) \cdot\left|Z_{\text {SINGLE_LISN }}\right|
$$

To experimentally show how the method works, we firstly installed the reference conducted emission setup with two LISNs (Fig.3) and thereafter installed the alternative conducted emission setup with 4 four LISNs (Fig. 4) and also with 6 LISNs (Fig.5), as it was shown in [6]. The mains simulation with four LISNs has one LISN pair in parallel for phase and one LISN pair for neutral in order to form different source impedance that simulates industrial mains environment but stable and without resonances. Similarly, the mains simulation with six LISNs has a trinity of three LISNs in parallel for phase and the other trinity of three LISNs for neutral in order to form different source impedance that simulates other industrial mains environment. The reason why we install the mains simulations by different configuration of LISNs can be simply explained as follows; the different configuration of LISNs as seen in Fig. 4 (and also in Fig. 5) yields a different CM and DM impedance from the reference LISN setup given in Fig. 3. This impedance is stable and without resonances so that it provides us with a good verification environment.

After the verification the EUTs were directly connected to mains without any LISN. Three types of EUTs (see Fig.6); a drill, an UPS and a reference source supplied with 220 VAC were used. The drill was emitting predominantly in DM and gave us opportunity to focus specially on DM emissions. Conversely, the reference

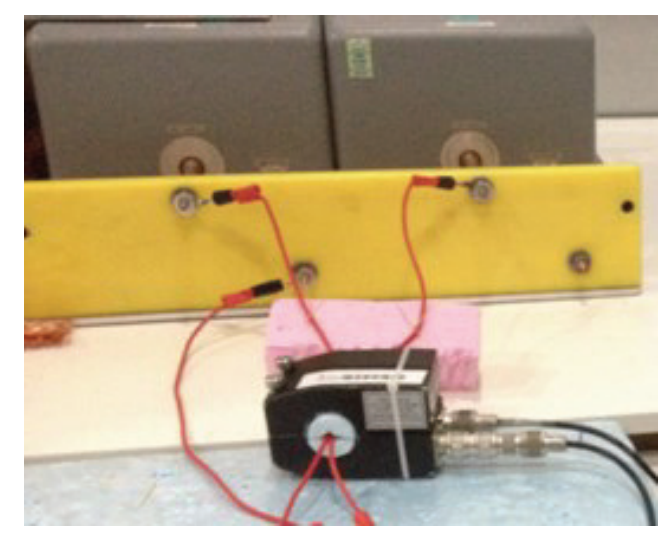

Fig 3. Reference experimental setup

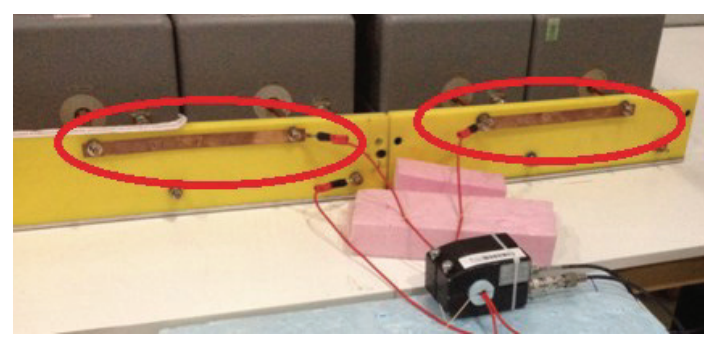

Fig 4. Mains simulation with 4 LISN

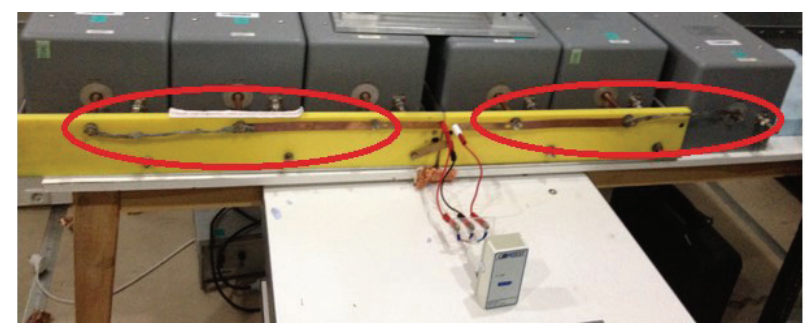

Fig 5. Mains simulation with 6 LISN

source supplied with 220 VAC was emitting in CM and it was the good example to specially study the CM emission. Finally, the UPS was emitting in both CM and DM so that we have the opportunity to study both of the emission type at the same time and to merge the results in order to calculate the final result expected to exist on the LISN RF port in the reference setup. In this method no Capacitive Voltage Probe (CVP) was used, the CM and DM voltages were calculated instead by multiplying the CM and DM currents with the corresponding $\mathrm{CM}$ and DM supply impedances in order to simplify the setups and to lower the measurement uncertainty. In addition, measuring CM and DM currents separately is significantly easier than separate $\mathrm{CM}$ and DM direct voltage measurements.

All the stated EUTs (see Fig.6) were measured in the reference setup, in the mains simulations with 4 LISNs and with 6 LISNs and also directly on the actual mains in turn. Thereafter, theoretical correction factors were calculated for comparison with the experimental factors to validate the proposed method. Ultimately, we combined the CM and DM voltages in order to link the measured currents in the alternative setups to the voltage expected on the RF port of LISNs in the reference setup. As the actual mains may be noisy, as a first precaution, we utilized one of the mains sockets of our laboratory as the actual mains. As the labo- 
ratory is equipped with some mains filters at the laboratory input, it partly helped us with obtaining a cleaner mains socket at some frequencies. On the other hand, also, we always made sure that measured emissions really came from the EUTs not from the mains, before starting the measurements. To simply do this, in the first place, we generally switched off the EUT and checked if the disturbance dropped at least by $10 \mathrm{~dB}$. Besides, in some cases, if required, the other effective way was to replace the EUT with a resistive load that draws the similar current (or a higher current) as the EUT and then, checked if the emissions were lower than the EUT emissions or not. Generally, background noise lower by at least $10 \mathrm{~dB}$ than the emitted signal from the EUT was regarded acceptable. In some cases in which the $10 \mathrm{~dB}$ criterion was not met (between $6 \mathrm{db}$ and $10 \mathrm{~dB}$ ), it slightly contributed to the measurement uncertainty and caused slight deviation between theoretical and experimental correction factors. When the background noise was less than $6 \mathrm{~dB}$ to the EUT emissions due to the fact that actual EUTs do not emit sufficiently or due to high supply impedance in some frequencies, these frequencies were removed from evaluation and analysis, as we need strong signals from the EUT in order for efficient comparisons between theoretical and experimental correction factors.

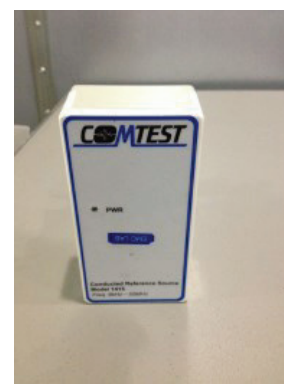

(a)

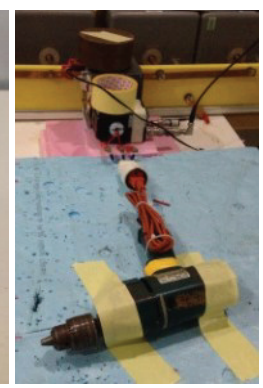

(b)

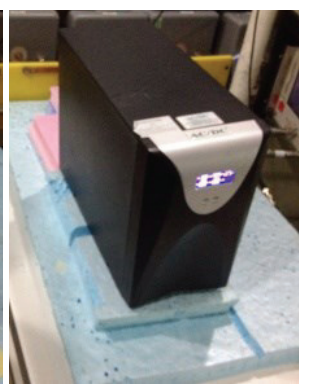

(c)
Fig 6. Used EUTs (a) a reference source, (b) a drill, (c) a UPS

\section{Experimental Results And Discussions}

As the first step, we tested the conducted reference source directly on the mains and linked the results to the LISN RF port of the reference setup in CM. Measurements and correction factors of this EUT (reference source) on mains simulations with 4 and 6 LISNs can be obtained from [6], now we only focus on the measurements of the reference source directly on the laboratory mains without any extra components. The CM impedance of the reference source is shown in Fig.7(a), actual mains in 7(b) and finally reference setup in Fig.7(c). The mains impedance seen in Fig.7 (b) is the instant snapshot and varies slightly and continuously. In Fig.7(c), the CM impedances of the mains simulations installed with four and six LISNs are also given just for information besides the reference LISN CM impedance. Because we use military LISNs, the CM impedance of the reference LISN system is around $22 \mathrm{ohm}$, as stipulated by the standard. The reference $\mathrm{CM}$ impedance increases slightly as the frequency approaches $30 \mathrm{MHz}$ because there is a buffer zone in front of the LISNs and it starts to come into play. The CM impedance of the actual laboratory mains shows a different behavior with severe resonances but it is less than $100 \mathrm{ohm}$ in the most of the frequency band analyzed. Now, as seen in Fig. 8 (a) and
Fig.8 (b), we can easily calculate the $\mathrm{K}$ correction factors that form the correlations between the reference setup and the laboratory mains by using the impedance curves given in Fig.7 (a) - 7(c) and the equations (9) - (10), and finally the LISN voltage expected to exist on the LISN in the reference setup is reached as given in Fig.8(c). The slight differences seen in Fig.8(a) and Fig.8(b) in lower frequencies between the theoretical and experimental correction factors are caused by the uncontrolled slight and continuous change of the mains impedance resonances in terms of level and frequency. The Fig.8(c) shows the comparison between the experimental measured voltage at the LISN RF port and the calculated LISN voltage, which is obtained by using (13), in the reference setup.

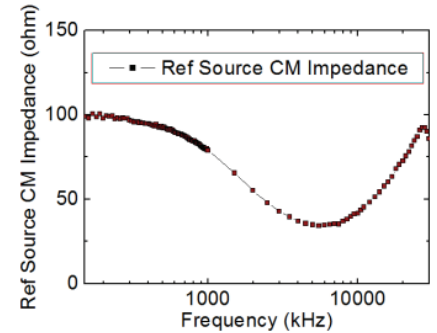

(a)

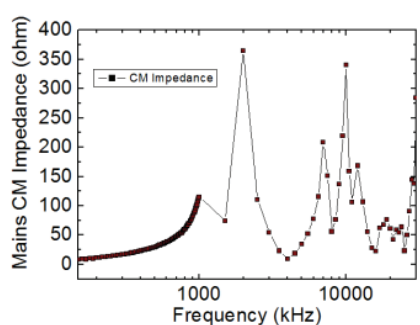

(b)

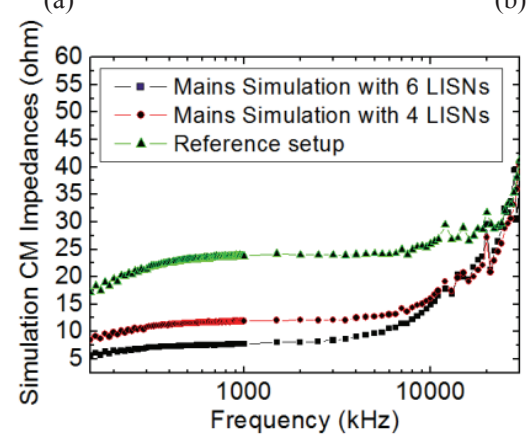

(c)

Fig 7. Results related to reference source on actual mains, (a) EUT CM impedance, (b) Mains CM impedance, (c) reference LISN CM impedance together with mains simulations installed with 4 and 6 LISNs

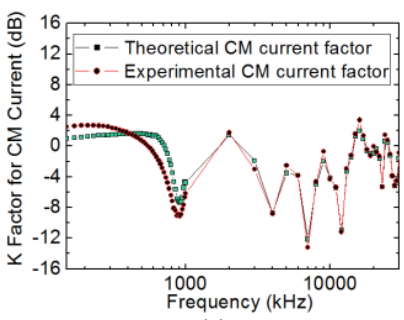

(a)

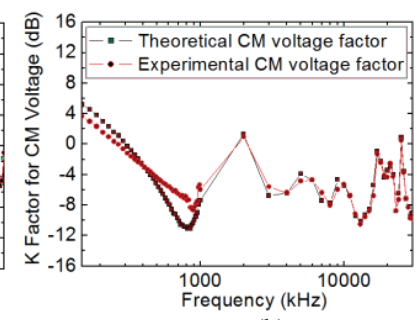

(b)

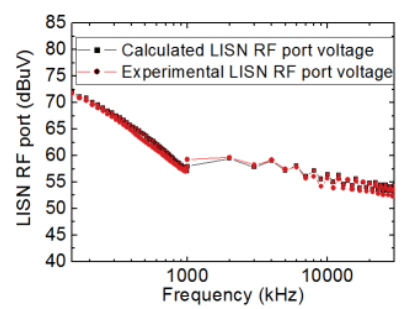

(c)

Fig 8. CM Mode correction factors for reference source in actual mains (a) current factors, (b) voltage factors, (c) LISN RF port voltage 


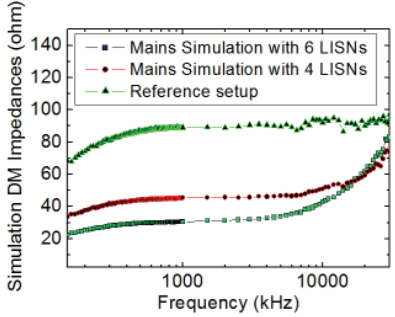

(a)

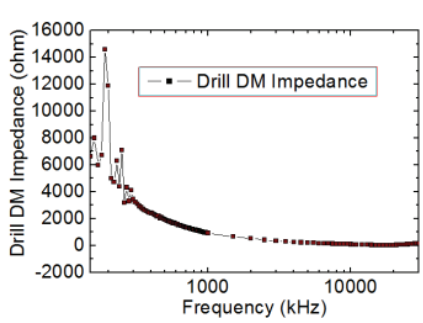

(b)
Fig 9. DM impedances (a) reference setup and mains simulations (b) drill impedance

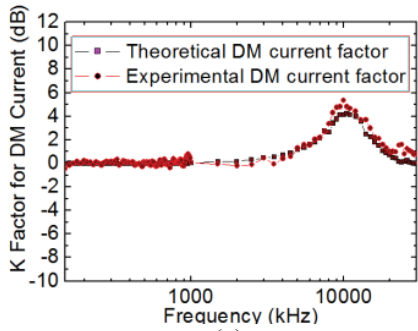

(a)

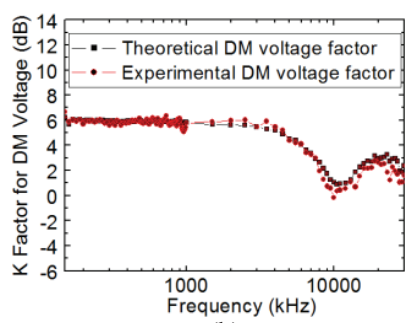

(b)
Fig 10. DM Mode correction factors for drill in mains simulation installed with 4 LISNs (a) current, (b) voltage

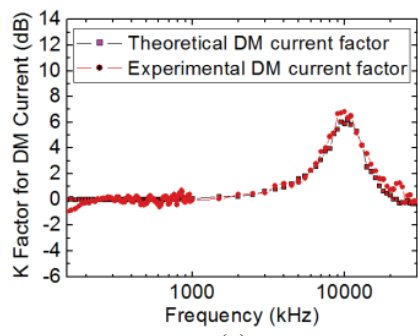

(a)

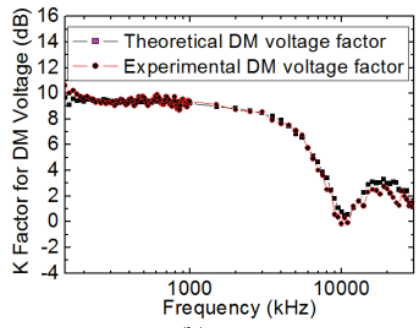

(b)
Fig 11. DM Mode correction factors for drill in mains simulation installed with 6 LISNs (a) current, (b) voltage

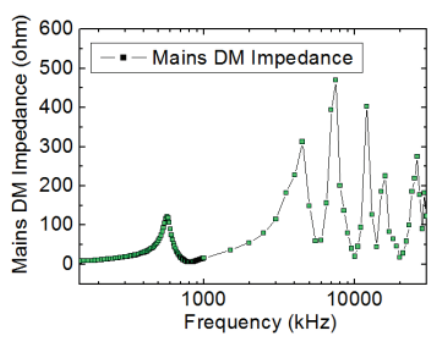

(a)

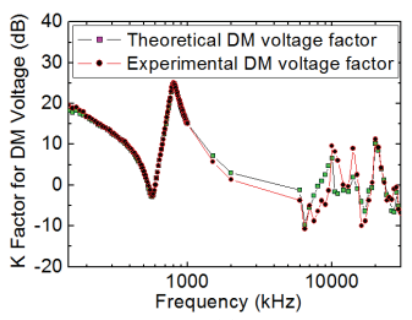

(c)

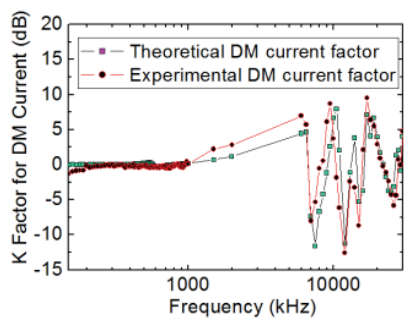

(b)

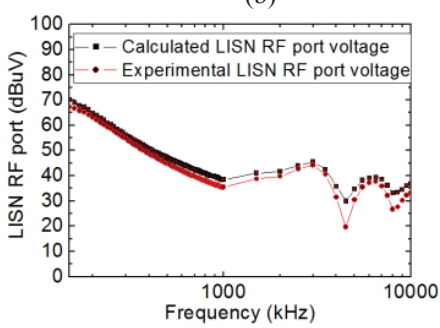

(d)
Fig 12. Results related to drill on actual mains (a) DM impedance of actual mains, (b) K current factors, (c) K voltage factors, (d) LISN RF port voltage

We continued the measurements with a drill supplied by 220 VAC. Since we detected that CM currents of the drill were negligible in comparison to DM currents, the CM current was neglected for the drill unlike the reference source. The drill was measured on the LISN simulations installed with 4 and 6 LISNs and also on the actual mains in turn. The DM impedance of the simulation mains installed with 4 and 6 LISNs and the impedance of the drill are presented in Fig.9. The EUT, a drill in our case here, has high DM impedance in the most of the frequency range and has huge resonances as depicted in Fig.9(b). The DM impedance of the reference LISN system installed with two LISNs as stipulated by the standard is around $90 \mathrm{ohm}$ since we used military LISNs in our research and the declared impedance of a LISN by the manufacturer is around $44 \mathrm{ohm}$. The reference DM impedance increases slightly as the frequency approaches $30 \mathrm{MHz}$ because there is a buffer zone in front of the LISNs and it starts to come into play. The DM impedance of the mains simulations installed with four LISN and six LISN show different behaviors from the reference impedance as seen in Fig.9(a). Now, we can easily calculate the K correction factors that form the correlations between the reference setup and the mains simulations by using the impedance curves as seen in Fig.9. In the first place, Fig.10 shows the comparison of the theoretically calculated correction factors based on the impedance measurements with the experimental correction factors for DM current and voltages for the mains simulation installed with four LISNs. Similarly, the results of the drill in the other mains simulation installed with 6 LISNs are given in Fig.11. All the results for the drill show that there is a very good agreement between the theoretical and experimental results in terms of correction factors. Ultimately for the drill, we tested it directly on the laboratory mains. The DM mains impedance of the actual laboratory mains is given in Fig.12 (a), the current and voltage $\mathrm{K}$ factors in Fig.12(b) - 12(c). Slight differences in some frequencies are again caused by uncontrolled instant changes in the DM mains impedance. Fig.12(d) shows the comparison between the experimental measured voltage at the LISN port and the calculated LISN voltage which is obtained by using (13), in the reference setup. The results of the drill again show a very good agreement between the experimental LISN results and the calculated results derived from impedance measurements.

As a complex EUT, we continued with the UPS on the mains simulations and on actual laboratory mains. The UPS was emitting in CM and DM so that we studied each circuit model separately and then merged the CM and DM models in order to calculate the final result expected to exist on the LISN RF port of the reference setup and finally compared it with the actual experimental value. The CM and DM impedances of the UPS in mains simulation are shown in Fig.13(a) - 13(b). The CM and DM impedances of the UPS show a very different behavior from each other. The CM/DM impedances of the reference LISN system and the impedances of mains simulations installed with four and six LISNs have been already given in Fig.7(c) and in Fig.9 (a). The K correction factors for CM/DM current/voltage, which form the correlations between the reference setup and mains simulations with four and six LISNs by using the impedance curves, are presented in Fig.14 - Fig.17 respectively. Fig.14 - Fig.17 also show a very good agreement between the theoretical and experimental $\mathrm{K}$ factors. Secondly for UPS, the $\mathrm{K}$ correction factors for CM/DM current/voltage that form the correlations between the reference setup and the actual mains are given in Fig.18 - Fig.19. Slight differences in the K factor measurements especially related to the actual mains are caused by uncontrolled instant changes in the mains impedance. The graphs given so far for UPS reveal that CM and DM emissions of 
an EUT can be easily modeled separately. The predicted LISN RF port voltage and experimental LISN RF port voltage are presented in Fig.20 as a final result. The overall chain of curves in the Fig.14 Fig. 20 also reveals that the current measured on the alternative setups can be linked to the maximum expected voltage measured on the LISN RF port in the reference setup. This chain can be explained in detail as follows; the CM and DM currents measured on the alternative setups are firstly linked to the CM and DM currents flowing in the reference setup by means of the $\mathrm{K}$ factors. Once the reference CM and DM currents are calculated by $\mathrm{K}$ factors, the maximum expected LISN voltage on one of the LISNs in the reference setup is predicted by using (13) as the worst case situation. In all the graphs of the research, while the theoretical corrections factors were calculated by using measured impedance values and using (9) - (12), the experimental correction factors were obtained by performing actual conducted current emission measurements in both of the reference and the alternative test setups in turn. As it is easily noticed in the graphs, the K factor graphs of drill and UPS have less number of frequency spots than the graphs of the reference source because the reference source emits in the full frequency range in a good level. On the other hand, the actual EUTs; the drill and the UPS do not emit in full frequency range or do emit low-level emissions that are intolerably close to background noise in some frequencies, which prevents efficient RF current measurements. For that reason, we had to use only the strong emitted frequencies or frequency ranges since we needed a good level of signals which are higher enough from the background noise in order for efficient comparisons between theoretical and experimental $\mathrm{K}$ factors. It should be also specially stated that the efficient RF current measurements were also hampered due to high impedance of the supply and the EUT as high impedance of the overall test circuit reduced the flowing current on the test circuit and the measurable current levels depend on the overall test circuit impedance value. For that reason, the numbers of analyzed spot frequencies or analyzed frequency ranges for mains simulations and the actual mains are sometimes different from each other especially in the measurements of the actual EUTs; the drill and the UPS.

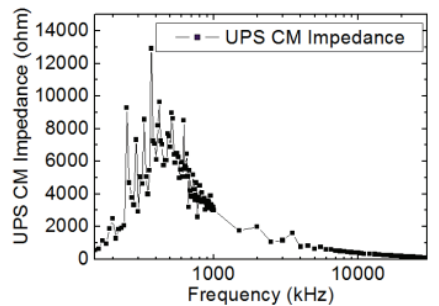

(a)

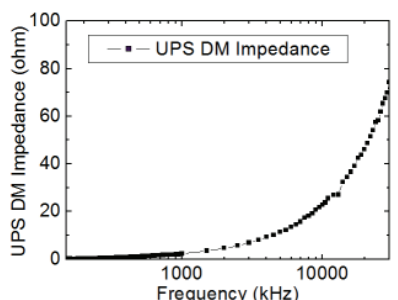

(b)
Fig 13. UPS impedances (a) CM, (b) DM

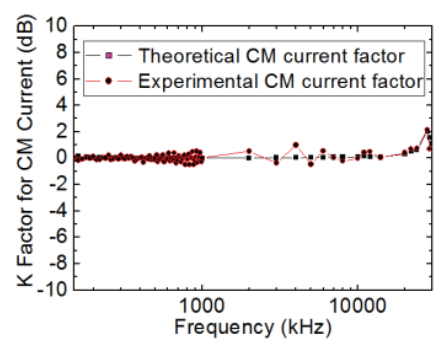

(a)

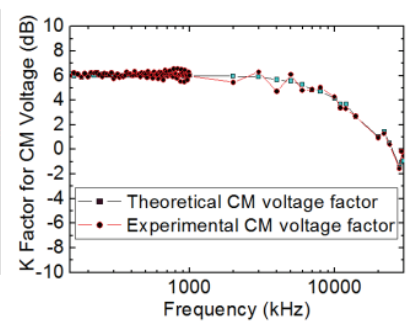

(b)
Fig 14. CM Mode correction factors for UPS in mains simulation installed with 4 LISNs (a) current factor, (b) voltage factor

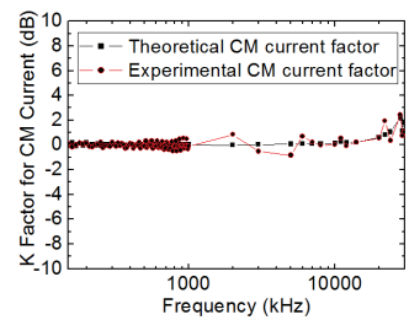

(a)

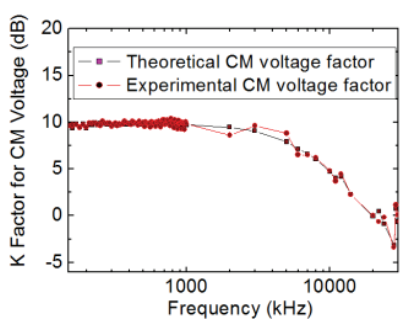

(b)
Fig 15. CM Mode correction factors for UPS in mains simulation installed with 6 LISNs (a) current factor, (b) voltage factor

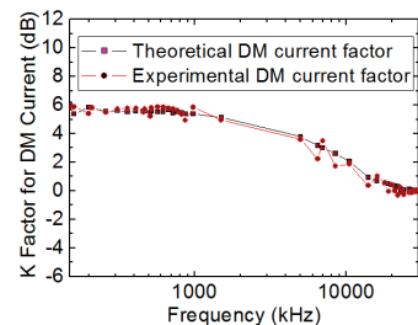

(a)

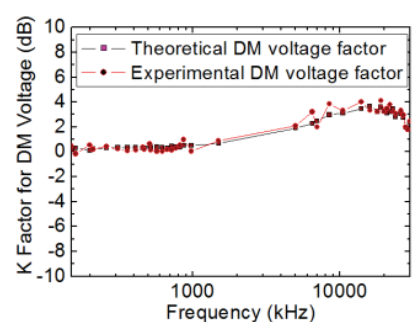

(b)
Fig 16. DM Mode correction factors for UPS in mains simulation installed with 4 LISNs (a) current factor, (b) voltage factor

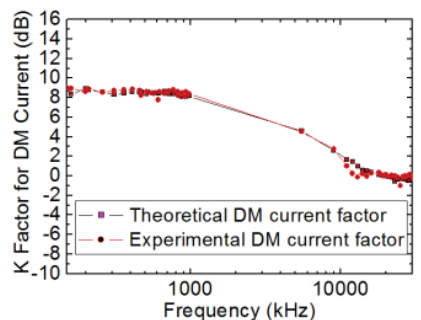

(a)

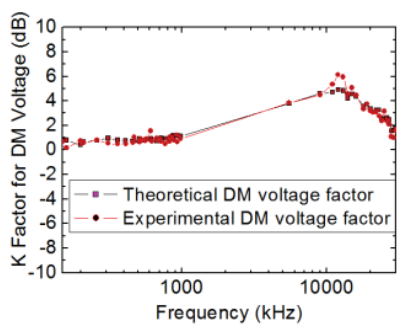

(b)
Fig 17. DM Mode correction factors for UPS in mains simulation installed with 6 LISNs (a) current factor, (b) voltage factor

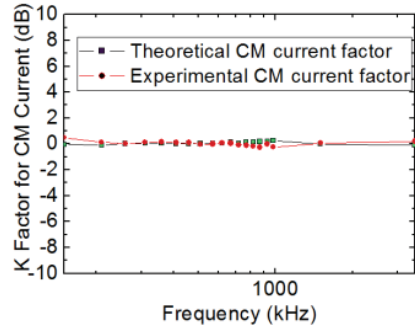

(a)

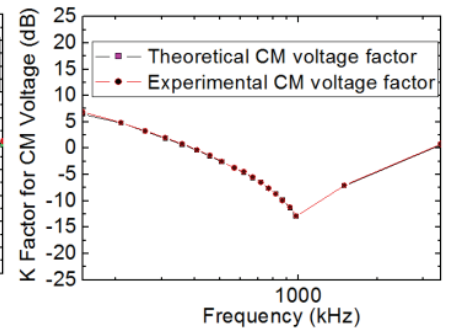

(b)
Fig 18. CM Mode correction factors for UPS on actual mains (a) current factor, (b) voltage factor

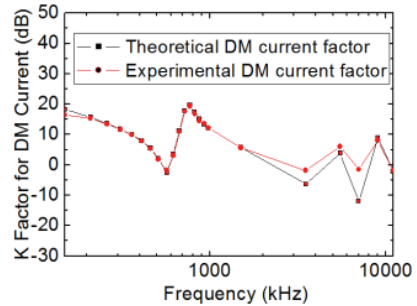

(a)

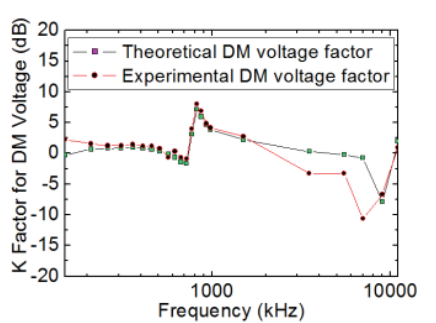

(b)
Fig 19. DM Mode correction factors for UPS on actual mains (a) current factor, (b) voltage factor 


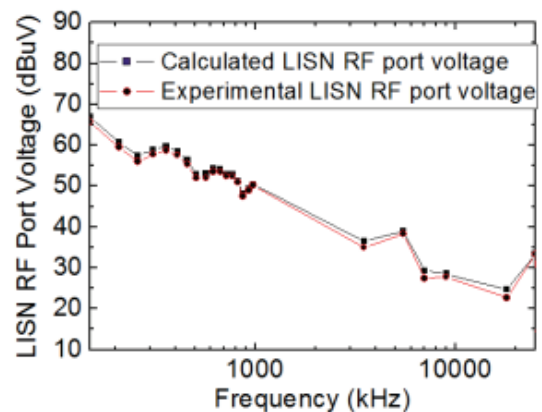

Fig 20. LISN RF port voltage; calculated result $(C M+D M)$ and experimental result

Finally, we checked the quality of the established link between the investigated alternative method and reference standard method by using GCEM Validation Tool (GVT) [8] for the final data figures; Fig.8(c) and Fig.12(d). As UPS do only emit in certain spot frequencies and the minimum 31 data points is required by the GCEM Validation Tool, Fig.20 was left out of the scope of this work. Table 1 shows the verdicts of the GVT as per various validation methods [9] for Fig. 8(c) and Fig.12(d).

Table.1 GVT GCEM Validation Tool Results

\begin{tabular}{|l|l|l|l|l|l|}
\hline & $\begin{array}{l}\text { FSV } \\
\text { (IEEE) }\end{array}$ & $\begin{array}{l}\text { FSV } \\
\text { (UPC) }\end{array}$ & IELF & R -Factor & $\begin{array}{l}\text { Correlati } \\
\text { on }\end{array}$ \\
\hline Fig. 8(c) & Excellent & Excellent & Excellent & Excellent & Excellent \\
\hline Fig.12(d) & Good & Excellent & Good & Excellent & Excellent \\
\hline
\end{tabular}

\section{Conclusion}

In this work, we brought our previous research further by using actual EUTs and also actual mains, and obtained reasonable results to predict emissions in reference setups when the EUT is tested in alternative locations. It was firstly shown that CM and DM circuit models can be separated for EUTs and each circuit can be focused separately in order to reach the correction factors that form correlation between alternative setups and the reference setup. Finally, the LISN RF port voltage of the reference setup can be reasonably predicted by using $\mathrm{K}$ correction factors and merging $\mathrm{CM}$ and $\mathrm{DM}$ emissions as the worst case.

\section{Acknowledgment}

This research is in the scope of the project IND60 "Improved EMC test methods in industrial environment" and financially supported by European Metrology Research Programme.

The authors thank Savas Acak for his technical support.

\section{References}

[1] CISPR22, Information technology equipment -Radio disturbance characteristics - Limits and methods of measurement

[2] CISPR11, Industrial, scientific and medical equipment - Radio-frequency disturbance characteristics - Limits and methods of measurement

[3] Catrysse J., Vanhee F. , Knockaert J., Hendrickx I., Beauvois, V, “In situ testing of large machines: Alternative methods for conducted emission measurements", Electromagnetic Compatibility, IEEE International Symposium on Electromagnetic Compatibility, Detroit, MI, pp. 1 - 6, 18-22 Aug. 2008
[4] L.Ran, J. C. Clare, K. J. Bradley, C. Christopoulos, "Measurement of Conducted Electromagnetic Emissions in PWM Motor Drive Systems Without the Need for an LISN", IEEE Transactions On Electromagnetic Compatibility, Vol. 41, No.1, Feb. 1999.

[5] S.Coets, V. Beauvois, J. Catrysse, W. Legros, "Indirect Method of Measurement for Conducted Emissions of High-Power Equipments", International Symposium on EMC, EMC Europe 2006, Barcelona, Sep. 2006.

[6] S.Cakir, O.Sen, M.Cinar, M. Cetintas, "Alternative Conducted Emission Measurements for Industry", EMC Europe 2014, Gothenburg, Sweden, pp. 10371042, 1-4 September 2014.

[7] Tarateeraseth V., Bo Hu ; Kye Yak See, Canavero F.G., "Accurate Extraction of Noise Source Impedance of an SMPS Under Operating Conditions", IEEE Transactions on Power Electronics, Vol. 21, No.1,pp. 111-117, Jan 2010.

[8] GCEM Validation Tool (GVT) Version 3.1 [online]. Available: http://www.upc. edu/web/gcem/?p=392\&lang=en

[9] GCEM Validation Tool (GVT) Version 3.1 Reference Guide, December 2013 